Ann. Biol. anim. Bioch. Biophys., I967, y (I), 59-6r.

NOTE

\title{
EFFET DE L'INGESTION D'HUILE DE SOJA CHAUFFEE SUR L'ELIMINATION URINAIRE DE LA THIAMINE CHEZ LE RAT EN CROISSANCE
}

\author{
B. POTTEAU, Évelyne BIETTE et M. LHUISSIER \\ Laboratoire d'Étude des Qualités biologiques des Aliments de l'Homme, \\ Centre national de Recherches zootechniques, 78 - Jouy-en-Josas
}

L'ingestion de corps gras soumis à des conditions de chauffage sévères peut se traduire par un abaissement de l'efficacité biologique de certains constituants de la ration, en particulier de diverses vitamines. Cette modification a été observée dans le cas de la pyridoxine et de la riboflavine (WitTing et al., 1957), de la vitamine A (REPORTER et Harris, I961), et de la vitamine E (Alfin-Slater, AUERbach et AFTERGOOD, I959).

Peu d'auteurs semblent s'être intéressés au cas de la thiamine. Seuls Raju, NarayaNa Rao et Rajagopalan, en I965, ont signalé que chez des rats en croissance qui reçoivent un régime contenant I5 p. Ioo d'huile d'arachide chauffée à $270^{\circ} \mathrm{C}$ pendant 8 heures, la teneur du foie en thiamine s'accroît moins fortement que chez des témoins qui reçoivent un régime contenant I5 p. Ioo d'huile fraîche. Mais cette différence pourrait s'expliquer, au moins en partie, par un fort abaissement de la quantité de nourriture consommée lorsque le régime renferme de l'huile chauffée.

Il nous a donc paru intéressant de déterminer la quantité de thiamine excrétée par la voie urinaire, chez le Rat recevant un régime équilibré où l'apport lipidique est constitué d'huile chauffée ou non.

\section{MÉTHODES EXPÉRIMENTALES E'T MATÉRIEL UTILISÉ}

Une huile de soja raffinée est chauffée à $275^{\circ} \mathrm{C}$ pendant 12 heures, dans un appareillage entièrement en verre. Pendant toute la durée de l'opération, l'huile est protégée d'une oxydation par l'air grâce à un barbotage d'azote. 
Quatre lots de dix jeunes rats Wistar, mâles, d'un poids moyen de $85 \mathrm{~g}$, reçoivent des régimes se différenciant selon l'apport lipidique :
lot $\mathrm{C}_{1}$ : ro p. roo d'huile chauffée,
lot $\mathrm{T}_{1}$ : Io p. Ioo d'huile fraîche,
lot $\mathrm{C}_{2}: 20$ p. roo d'huile chauffée,
lot $T_{2}: 20$ p. 100 d'huile fraîche.

Ces régimes ont la composition suivante :

- constituants non vitaminiques, en $\mathrm{g}$ par $\mathrm{kg}$ : caséine $\mathrm{I} 80$, huile de soja chauffée ou non roo ou 200, saccharose 200 (régime à 20 p. 100 d'huile) ou 300 (régime à Io p. d'huile), amidon 370 , mélange salin de HuBBEL et al. sans calcium 20, carbonate de calcium ro, agar-agar 20.

- constituants vitaminiques, par $\mathrm{kg}$ : vitamine $\mathrm{A} 2000 \mathrm{UI}, \mathrm{D}_{2} 500 \mathrm{UI}$, acétate de DL. $\alpha$-tocophérol $15 \mathrm{mg}$, vitamine $\mathrm{K}_{1} \mathrm{I} \mathrm{mg}$, thiamine $4 \mathrm{mg}$, riboflavine $4 \mathrm{mg}$, pyridoxine $4 \mathrm{mg}$, amide nicotinique $50 \mathrm{mg}$, pantothénate de calcium ro $\mathrm{mg}$, choline $500 \mathrm{mg}$, inositol $200 \mathrm{mg}$, acide folique I mg, acide $p$-amino-benzoïque $500 \mathrm{mg}$, biotine $0,2 \mathrm{mg}$, vitamine $\mathrm{B}_{12} 0,03 \mathrm{mg}$.

Après avoir été soumis à ces régimes pendant 17 jours, les animaux atteignent les poids moyens suivants :

$$
\begin{aligned}
& -\operatorname{lot} \mathrm{C}_{1}: 166 \mathrm{~g}, \\
& -\operatorname{lot} \mathrm{T}_{1}: 188 \mathrm{~g}, \\
& -\operatorname{lot} \mathrm{C}_{2}: 108 \mathrm{~g}, \\
& -\operatorname{lot} \mathrm{T}_{2}: 159 \mathrm{~g} .
\end{aligned}
$$

A ce stade, les animaux sont installés, pendant une durée d'une semaine, dans des dispositifs de bilans permettant la récolte séparée des urines et des fèces. Les urines sont recueillies sur acide acétique. Les consommations de nourriture, les gains de poids corporels et les quantités de thiamine excrétées par l'urine sont déterminées pendant cette période. Le dosage de la vitamine est effectué par voie microbiologique, en utilisant Lactobacillus viridescens cultivé sur milieu Difco.

\begin{tabular}{|c|c|c|c|c|}
\hline & $\begin{array}{l}\text { Consommation } \\
\text { de nourriture } \\
\text { (g par jour) }\end{array}$ & $\begin{array}{c}\text { Gain } \\
\text { de poids } \\
\text { (g par jour) }\end{array}$ & $\begin{array}{l}\text { Thiamine } \\
\text { urinaire } \\
\text { ( } \mu \text { g par jour) }\end{array}$ & $\frac{\text { Thiamine urinaire }}{\text { Thiamine ingérée }} \times 100$ \\
\hline $\begin{array}{l}\text { Lot } C_{1} \ldots \ldots \ldots \ldots \ldots \ldots \ldots \\
\text { Lot } T_{1} \ldots \ldots \ldots \ldots \ldots \ldots \\
\text { Signification des différ. }\end{array}$ & $\begin{array}{l}15,9 \pm 0,3 \\
15,5 \pm 0,6 \\
\text { NS. * }\end{array}$ & $\begin{array}{l}3,8 \pm 0,2 \\
3,8 \pm 0,3 \\
\text { NS. * }\end{array}$ & $\begin{array}{r}5,0 \pm 0,2 \\
10,0 \pm 0,8 \\
P<0,01^{* *}\end{array}$ & $\begin{array}{c}7,8 \pm 0,4 \\
16,1 \pm 1,1 \\
\mathrm{P}<0,01 * *\end{array}$ \\
\hline $\begin{array}{l}\text { Lot } \mathrm{C}_{2} \ldots \ldots \ldots \ldots \ldots \ldots \ldots \ldots \ldots \\
\text { Lot } \mathrm{T}_{2} \ldots \ldots \ldots \ldots \ldots \ldots \\
\text { Signification des différ. }\end{array}$ & $\begin{array}{l}10,4 \pm 0,5 \\
13,8 \pm 0,5 \\
\mathrm{P}<0,01^{* *}\end{array}$ & $\begin{array}{c}2,7 \pm 0,2 \\
4,8 \pm 0,4 \\
\mathrm{P}<0,01 * *\end{array}$ & $\begin{array}{r}2,7 \pm 0,3 \\
5,7 \pm 0,2 \\
\mathbf{P}<0,01 * *\end{array}$ & $\begin{array}{c}6,4 \pm 0,8 \\
10,4 \pm 0,5 \\
\mathrm{P}<0,01 * *\end{array}$ \\
\hline
\end{tabular}

\section{RÉSULTATS}

Les résultats obtenus pendant cette dernière semaine sont les suivants (moyenne \pm erreur type) :

* Différences non significatives.

** Différences significatives au seuil de P.

Pour un taux de Io p. Ioo d'huile fraîche ou chauffée dans les régimes, les quantités de nourriture consommées par les animaux et leurs gains de poids corporels ne diffèrent pas de façon significative. Par contre, pour un taux de $20 \mathrm{p}$. Ioo d'huile 
dans leur régime, les animaux qui ingèrent l'huile chauffée consomment moins de nourriture et ont une croissance ralentie par rapport aux témoins qui reçoivent l'huile fraîche.

L'élimination urinaire moyenne de thiamine est abaissée de façon très significative chez les animaux dont le régime contient Io ou 20 p. Ioo d'huile chauffée par rapport à celle des témoins correspondants. Cette différence est encore très marquée lorsque les excrétions sont rapportées aux quantités de vitamine ingérées : il ne semble donc pas que, dans le cas du $\operatorname{lot} \mathrm{C}_{2}$, l'abaissement de l'excrétion urinaire de thiamine s'explique uniquement par la faible consommation de nourriture.

\section{CONCLUSION}

Nos résultats complètent ceux de Raju, Narayana RaO et RajagopalaN (I965). Ils peuvent s'interpréter de deux manières :

- ou bien l'ingestion de certaines huiles chauffées abaisse l'efficacité biologique de la thiamine, cet effet pouvant se situer au niveau digestif ou au niveau tissulaire ;

- ou bien elle augmente le besoin en thiamine pour une raison métabolique qu'il resterait à préciser.

Reçu pour publication en février 1967.

\section{REMERCIEMENTS}

Nous remercions M. FÉRon, Directeur technique de la Société Astra Calvé qui nous a fourni l'huile de soja raffinée utilisée pour ce travail.

\section{SUMMARY}

\section{EFFECTS OF INGESTION OF HEATED SOYBEAN OIL ON EXCRETION} OF THIAMINE IN URINE BY GROWING RATS

Soybean oil heated at $275^{\circ} \mathrm{C}$ for $\mathrm{I} 2$ hours with bubbling through nitrogen was given to growing rats as 10 or $20 \mathrm{p}$, Ioo by weight of a balanced diet. In those animals excretion of thiamine in urine was much less than in controls given unheated soybean oil in the same conditions.

\section{RÉFÉRENCES BIBLIOGRAPHUQUES}

Alfin-Slater R. B., Auerbach S., Aftergood L., 1959. Nutritional evaluation of heated oils. J.amer. Oil Chem. Soc., 36, 638-64I.

RajU N. V., Narayana RaO M., RaJagopalan R., I965. Nutritive value of heated vegetable oils. J.amer. Oil Chem. Soc., 42, 774-776.

REPorter M. C., HarRis R. S., I96r. Effects of oxidized soybean oil on the vitamin A Nutrition of the Rat. J. amer. Oil Chem. Soc., 38, 47-51.

Witring L. A., Nishida T., Jornson O. C., Kummerow F. A., I957. The relationship of pyridoxine and riboflavin to the nutritional value of polymerized fats. $J$. amer. Oil Chem. Soc., 34, 42I-424. 